\title{
The role of meaning and familiarity in mental transformations
}

\author{
WENDY SMITH and ITIEL E. DROR \\ Southampton University, Southampton, England
}

\begin{abstract}
Eighty-four participants mentally rotated meaningful and meaningless objects. Within each type of object, half were simple and half were complex; the complexity was the same across the meaningful and meaningless objects. The patterns of errors were examined as a function of the type of stimuli (meaningful vs. meaningless), complexity, and angle of rotation. The data for the meaningful objects showed steeper slopes of rotation for complex objects than that for simple objects. In contrast, the simple and complex meaningless objects showed comparable increases in error rates as a function of angle of rotation. Furthermore, the slopes remained comparable after pretraining that increased familiarity with the objects. The results are discussed in terms of underlying representations of meaningful and meaningless objects and their implications to mental transformations. The data are consistent with a piecemeal rotation of the meaningful stimuli and a holistic rotation of the meaningless stimuli.
\end{abstract}

Mental rotation, like the physical rotation of an object, requires more time to complete as the degree of rotation becomes greater. Mental imagery also resembles perception, in that information about the spatial relations is preserved within the representation (see Kosslyn, 1981, for a detailed explanation). Image rotation can be understood, within this context, as a series of transformations of mental iconic representations. However, it is still unclear whether mental transformations are holistic or piecemeal. In a holistic process, the entire image is rotated as a single entity. In contrast, in a piecemeal process the image is rotated segment by segment, the "whole" being reestablished through the spatial relations among the segments.

Previous research has focused on whether response time data show an interaction between the complexity of the images and angular rotation. Cooper and Podgorny (1976) argued that if an image is transformed holistically, the entire image would be rotated as a single unit, and, therefore, the complexity of the image should not affect the rate of rotation (for a detailed account of this logic, see Cooper \& Podgorny, 1976). Using polygons of varying complexity, Cooper and Podgorny found that the rate of rotation was not affected by the complexity of the stimuli and concluded that a holistic process underlined visual mental rotation. However, other researchers have found conflicting evidence; in

This article was supported by ESRC Student Grant R00429634239 awarded to W.S., and by a United States Air Force Research Laboratory grant awarded to I.E.D. The authors thank Christine Weston and Vered Rafaely for their help in assessing the stimuli, and John Wixted, Michael Corballis, Apostolos P. Georgopoulos, and an anonymous reviewer for valuable comments on earlier drafts of the paper. Correspondence concerning this article should be addressed to W. Smith, Department of Psychology, University of Southampton, Highfield, Southampton, SO17 1BJ, England (e-mail: ws@ soton.ac.uk). their studies, image complexity affected the rate of rotations (e.g., Folk \& Luce, 1987; Yuille \& Steiger, 1982).

A second approach in investigating the nature of visual mental rotation is to use hierarchically structured stimuli of large letters composed of smaller letters (Robertson \& Palmer, 1983). Participants had to rotate the stimuli and assess whether at least one aspect of the letters (either the large letter or the small letters) was normal or mirrorreversed. Robertson and Palmer found an advantage when the task was based on the larger letters and concluded that rotation is a holistic process. However, this paradigm has also been inconclusive. For example, Paquet (1991), using similar tasks and stimuli, showed that the data could be explained equally well as a piecemeal process.

A third approach for exploring the nature of image rotation is to compare the rotation slopes of possible and impossible objects (Dror, Ivey, \& Rogus, 1997). Impossible objects are more difficult to represent and process holistically than are possible objects (Schacter, Cooper, \& Delaney, 1990; Schacter, Cooper, Delaney, Peterson, \& Tharan, 1991). Dror et al. reasoned, therefore, that impossible objects are easier to transform in a piecemeal process than in a holistic one. According to this logic, if the slope of rotation of impossible and possible objects were comparable, this would suggest that possible objects were rotated in a piecemeal fashion, just like the impossible objects. Alternatively, if the slope of rotation were different, this would suggest that the possible objects were rotated holistically. Dror et al. found that the rates of rotation of the possible objects were identical to those of the impossible objects and concluded that, when possible, a piecemeal process underlined visual mental rotation. However, the results of Dror et al. demonstrated only that possible and impossible objects were rotated using the same process; the conclusion that this process was piecemeal rests 
on the assumption that the impossible objects were not represented and rotated holistically.

The research reported here stems from the belief that the initial representations play an important role in determining the nature of subsequent processing (Ashworth \& Dror, 2000; Dror \& Gallogly, 1999; Dror, Zagaeski, \& Moss, 1995; Marr, 1982). In the context of this study, we wanted to investigate whether the meaningfulness of the stimuli entailed different formats of representations with a subsequent difference in mental processing and pattern of results.

The present study differs from previous rotation research because error rates were used as the primary dependent measure rather than response time. Performance levels were defined according to speed and accuracy of response. Past research has shown that error rates reflect rotation in a comparable way to response times and are to some extent interchangeable (e.g., Dror et al., 1997; Dror \& Kosslyn, 1994; Salthouse, 1992). However, error rates can also be a unique dependent measure, providing additional insights into the nature of the visual mental rotation that response times cannot.

Cooper and Podgorny (1976) suggested that two factors contribute to response times during mental rotation. First, response times increase with greater angular distance through which the representation needs to be rotated. This will be found in both holistic and piecemeal representations. Second, response times increase with a greater number of segments that need to be processed. In a holistic representation, only one segment is rotated, whereas in a piecemeal representation, more segments are rotated, and the number of segments in the piecemeal representation increases with greater complexity of the object. Following this logic, holistic representations would not entail greater response times with increased complexity, but piecemeal representations would show such an effect. Therefore, a piecemeal representation would produce an interaction between angle and complexity (because both factors influence response times), and a holistic representation would produce no interaction (because only the angle contributes to the response times for the rotation). However, a pattern of results with no interaction between angle of rotation and complexity could also be found with a piecemeal representation. For example, if the segments in a piecemeal representation were processed in parallel, rather than one at a time, the resulting response times may not increase with the complexity of the objects. The pattern of results would then be identical to that predicted by a model of mental rotation that uses a holistic representation.

The limitations associated with the use of response times as a dependent variable can be dealt with by using error rates as the primary measure. Holistic and piecemeal rotation processes predict distinct patterns of errors. As with response times, one needs to distinguish between two sources of error in mental rotation. The first source is the angle of rotation. As the image is rotated greater distances, more processing is required. More errors occur as pro- cessing demands increase (e.g., older people have reduced processing capacity and, thus, have a more notable increase in this source of error; see Cerella, Poon, \& Fozard, 1981; Dror \& Kosslyn, 1994). This source of error will affect both the holistic and piecemeal rotation processes, and hence, they both predict a main effect of angle of rotation; that is, error rate will increase with greater angular rotations. This source of error mirrors that of the response time measure.

In contrast to the first source of error, the second source of error provides possibilities that distinguish underlying representations in rotation that response times do not. The second source of error is directly dependent on, and a function of, the particular type of transformation involved in the rotation process. This difference is due to the way that the spatial relations are represented within the image. In a holistic representation, the spatial relations are an integral part of the representation, and the entire image is effectively one unit. In a piecemeal representation, the spatial relations are a distinct part of the representation, with the segments retaining a greater level of independence. When the rotation is a piecemeal process, it involves moving the different segments of the image, one by one, and realigning them to maintain the proper spatial relations among them. The errors in this type of movement are dependent on the number of segments in the representation; more errors will occur in complex objects with a larger number of segments. However, this will also be predicted by a parallel transformation of many parts. Here, although the segments would be moved together, rather than one by one, the segments are still independent and need to be restored into the correct configuration during the rotation.

The holistic rotation mechanism does not encompass this source of error because there is only one segment to be affected, regardless of complexity. Of the two types of processing, therefore, only a piecemeal process will produce an interaction between the complexity of the rotated objects and angle of rotation. That is, the rate of increased errors with greater angular rotations will be a function of the number of segments in the image. In a holistic process, no such interaction is predicted; that is, the rate of errors as a function of angle of rotation will not be mediated by image complexity. One advantage of error rates over response times is that more errors can be expected with an increased number of segments, regardless of whether those segments are processed in parallel or not.

To summarize, the aim of this study is twofold. First, we examined whether mental rotation is a holistic or a piecemeal process by using the pattern of error rates. Second, we assessed how meaning mediates the representation of objects and their processing.

\section{EXPERIMENT 1 Meaningful Objects}

Meaningful objects were the stimuli in the first visual mental rotation experiment. Half the stimuli were rela- 
tively complex, and the other half relatively simple. It was predicted that, if meaningful images were rotated holistically, the rate of rotation for the simple and complex images would be comparable; if, however, they were rotated in a piecemeal fashion, one would find an interaction between the angle of rotation and complexity of the objects.

\section{Method}

Participants. The participants were 28 undergraduate students, who volunteered to participate in this experiment. There were 8 males and 20 females, who ranged in age from 18 to 33 (mean age, 20.9). All the participants had normal or corrected-to-normal vision and were right-handed. None of the participants had any experience with the stimuli or task prior to the experiment.

Materials. Two stimulus sets were used for this experiment; one was of six relatively complex objects, and the other was of six relatively simple objects (see Figure 1 for examples). Two additional objects were made for practice purposes only. First, all the upright objects were produced. This was achieved by producing line drawings of familiar objects, modified from templates available for artwork (Verso, 1988). All the drawings were formed from squares and rightangled triangles (half a square); each square measured $0.5 \times 0.5 \mathrm{~cm}$. The distinction between the simple and complex images was based on a variation of a compactness value that has been established by previous studies (e.g., Cooper \& Podgorny, 1976; Podgorny \& Shepard, 1983). The first measurement was the number of angles, or points, contained in the outline (Attneave, 1957; Cooper \& Podgorny, 1976) along with the number contained within the objects. The second measurement reflected the area of the object in terms of the number of $0.5-\mathrm{cm}$ squares in the object. The overall compactness value was calculated by taking the square root of the area measurement and dividing it by the first measurement (from Podgorny \& Shepard, 1983; see also Attneave, 1957). Lower compactness values indicated more complex objects. This measure revealed a significant difference be- tween the simple and complex objects. The mean compactness value was 0.17 for the complex objects $(S D=0.03$, range, $0.13-0.20)$ and $0.40(S D=0.17$, range, $0.22-0.68)$ for the simple stimuli $[t(10)=$ $3.43, p<.05]$.

The semantic categories to which the simple and complex objects belonged were matched as far as possible (e.g., simple object: ship; complex object: helicopter; matched through the semantic category of transport). Prior to using the stimuli, they were validated by conducting a preexperimental stimulus assessment evaluation in which 14 participants rated familiarity, meaningfulness, and representativeness across the stimulus types.

The next stage was to produce modifications for the stimuli to be used for the different trials. This was achieved by moving a small part of the stimulus to an adjacent location, avoiding any particularly salient features. For each stimulus, four modifications were made, each at a different part of the object.

Finally, each of the original stimuli and their four modifications were rotated clockwise in the picture plane to produce stimuli at $0^{\circ}$, $50^{\circ}, 100^{\circ}$, and $150^{\circ}$. Rotations of $180^{\circ}$ or larger were not selected in order to avoid ambiguity over whether to rotate clockwise or anticlockwise. In addition, this would discourage "flipping," or rotating in the depth plane (Murray, 1997).

The stimuli were used to construct two tasks; one task contained only the simple stimuli, and the other task contained only the complex stimuli. Each task consisted of 192 trials: six stimuli, each presented eight times at the four different orientations. The eight different presentations of each stimulus at each orientation included four same trials (unmodified stimuli) and four different trials (each one containing one of the four different modified versions). The total of 192 trials was organized into eight blocks of 24 trials each. Each block of trials included four presentations of each stimulus, once at each of the four angles of rotation. Two of the presentations of each stimulus were same trials and two were different trials, giving a total of 12 same and 12 different trials within each block. The trials in each block were organized so that the number of trials in each orientation
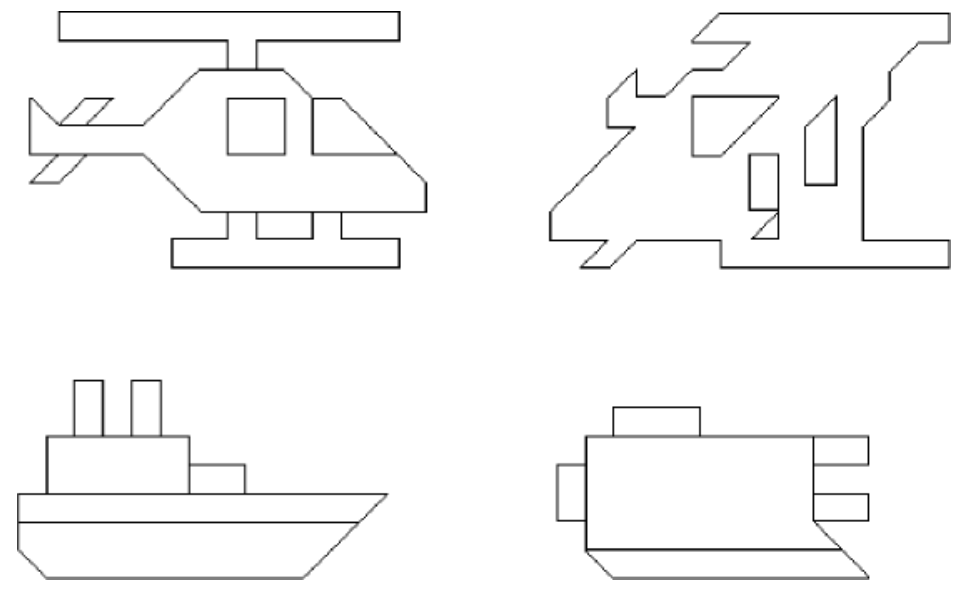

Figure 1. Examples of stimuli used in Experiments 1 and 2. The two objects on the left are examples of meaningful stimuli used in Experiment 1; the two objects on the right are examples of meaningless objects used in Experiments 2 and 3 . The meaningless objects were constructed from components of the meaningful objects. The meaningless object on the top right was constructed from the meaningful object on the top left; similarly, the object on the bottom right was derived from the object on the bottom left. The top two objects are examples of complex stimuli, and the bottom two objects are examples of simple stimuli. The complexity of the objects was measured by a compactness value and was matched across meaningful and meaningless stimuli. 
was equal and that half of the trials in each orientation were same and half were different. The trials within the eight experimental blocks were randomized.

The stimuli were presented using a Power Macintosh 8600/250 computer with a 17-in. Apple Vision monitor (running at 1,024 $\times 768$ resolution). The experiment was designed and administered using the commercial software Superlab, version 1.4 (Cedrus Corporation, 1991).

Procedure. Each participant performed the two experimental tasks; the tasks were identical in all respects, except that they used a different set of stimuli, either complex or simple. The order of administering the tasks was counterbalanced.

In each trial, the word "ready" was presented on the screen, and remained until the participant pressed the space bar with their nondominant hand. Following the space bar press a blank screen was presented for $500 \mathrm{msec}$, and then an upright version of a nonmodified object appeared on the screen. This object remained on the screen until the participant was ready to proceed. The participants were instructed to study this image carefully and only then to press the space bar. A mask would then appear for $500 \mathrm{msec}$. The mask was followed by the second object. This object was either exactly the same as the first object or was one of the modifications of it, and was presented at one of the four angles. It remained on the screen for $750 \mathrm{msec}$. After this, the words "same or different?" appeared on the screen. If the participant thought the two images were identical, regardless of orientation, they were instructed to respond by pressing the key labeled "same" (the "b" key on a standard keyboard). If they thought the two images were different from one another, they were to press the key labeled "diff" (the " $n$ " key).

The participants were instructed to respond immediately when they were prompted by the computer - that is, when "same or different?" appeared on the screen. Following their response, a blank screen appeared for $750 \mathrm{msec}$, and a new trial began with the word "ready" appearing on the computer screen.

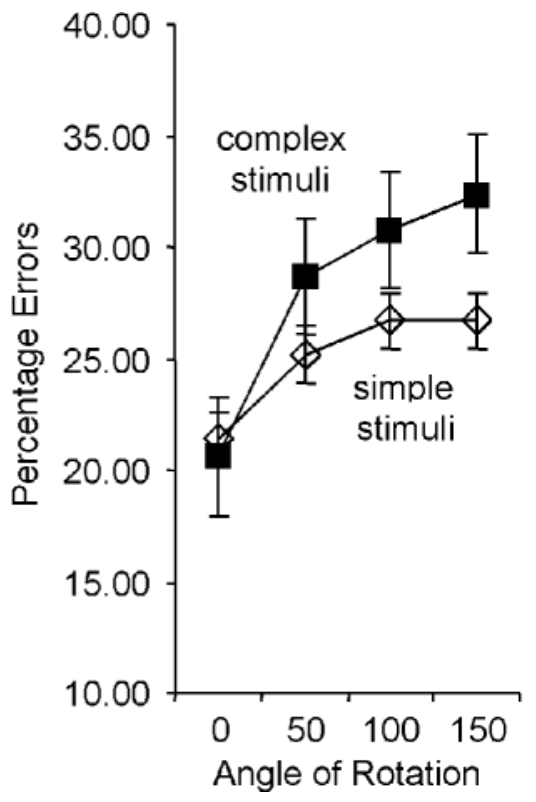

Prior to performing the experimental task, the participants completed a set of practice trials using objects that were not included in the actual experimental task. The practice trials were used to familiarize the participants with the instructions and procedures and to help them understand what constituted same and different images. Feedback was given during the practice, but not during the main experimental task.

\section{Results}

The error rates were submitted to analyses of variance (ANOVAs). Stimulus complexity (simple and complex) and angle of rotation $\left(0^{\circ}, 50^{\circ}, 100^{\circ}\right.$, and $\left.150^{\circ}\right)$ were used as within factors in the analyses.

First, the participants made increasingly more errors at greater angular rotations, as reflected by the main effect for angle of rotation $[F(3,81)=20.07, p<.01]$. Both the simple $[F(3,27)=5.37, p<.05]$ and the complex $[F(3,27)=$ $25.53, p<.001]$ objects showed an increase in errors with increasing angle. Second, as is illustrated in Figure 2 (left panel), there was a significant interaction between angle of rotation and stimulus complexity $[F(3,81)=4.89$, $p<.01]$. This reflected the fact that the rate of increased errors with greater angular distances was different for the simple and complex stimuli-specifically, that the rotation rate for the complex stimuli was steeper than that of the simple stimuli. Finally, there was a main effect of complexity $[F(1,27)=12.03, p<.01]$. There were more errors made with the complex stimuli than with the simple stimuli. There was no main effect of order (i.e., simple stimuli first and then complex or vice versa; $F<1) .{ }^{1}$

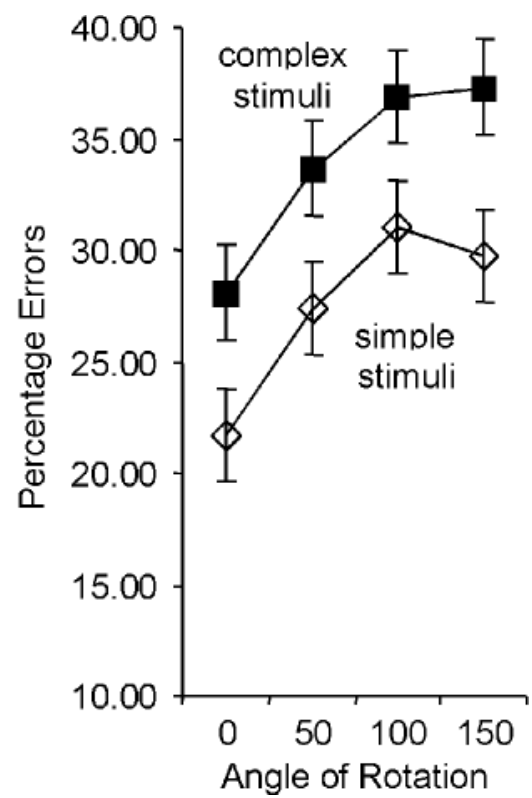

Figure 2. Error rate data for mental rotation of meaningful objects (left panel) and meaningless objects (right panel). For the meaningful objects, the slope of increased errors with greater angular rotation was different for the simple and complex objects, consistent with rotation using a piecemeal process. In contrast, the slope of increased errors with greater angular rotation for the simple and complex meaningless objects was identical, consistent with rotation using a holistic process. 
In the initial data analyses, same and different trials were grouped together, which may encompass both the decision process and a bias for responding. To avoid any such noise, the data were converted to $d^{\prime}$ values and reanalyzed. The additional analyses produced the same pattern of results. There was a main effect of angle of rotation $[F(3,81)=21.54, p<.001]$ and a significant interaction between angle of rotation and complexity $[F(3,81)=$ $8.08, p<.001]$. There was also a main effect of complexity $[F(3,27)=7.67, p<.05]$.

The response time data were analyzed to ascertain that a speed-accuracy tradeoff did not exist between errors and response time. Response times showed a main effect of angle $[F(3,81)=26.39, p<.001]$. This was present for both the simple $[F(3,27)=19.29, p<.001]$ and the complex $[F(3,27)=11.81, p<.001]$ objects. The response times increased with each successive angle of rotation, although, possibly due to the deadline procedure, this was only significant from $0^{\circ}$ to $50^{\circ}$ [complex: $t(27)=4.94, p<$ .001 ; simple: $t(27)=4.5, p<.001]$. There was a main effect of complexity $[F(3,27)=9.81, p<.05]$. The participants required more time to respond to complex objects than to the simple objects. There was no interaction between complexity and angle of rotation $(F<1)$. Hence, the analysis of the response time data ruled out a speedaccuracy tradeoff.

To ensure that the findings were not affected by variations in the study time of the stimuli with different complexity, the length of study of the simple and complex stimuli was examined. The study time was comparable in the two conditions $[t(27)=1.0, p>.05]$.

\section{Discussion}

This experiment examined the type of transformation used when the participants were mentally rotating meaningful objects. The increasing error rates as the angle of rotation increased reflects greater demand and use of visual mental rotation by the participants.

The important finding was that the rotation of the complex objects entailed a steeper slope of increasing errors as a function of angle of rotation than that for rotating the simple objects. This interaction is consistent with predictions made by a model that assumes that the images were rotated in a piecemeal process. If the rotation had been holistic, there would be no differences predicted in the slopes for rotating the simple and complex stimuli. These findings provide converging support for some studies that used response time as their measure (e.g., Folk \& Luce, 1987; Yuille \& Steiger, 1982) and with other studies that used a different experimental paradigm altogether (Dror et al., 1997; Paquet, 1991). However, the findings conflict with those of Cooper and Podgorny (1976), Robertson and Palmer (1983), and others.

The question that remains to be addressed is whether the pattern of results in this experiment was a function of the meaningfulness of the stimuli. To answer this question, we investigated in the next experiment how meaningless objects are represented and rotated in mental imagery.

\section{EXPERIMENT 2 Meaningless Objects}

The results of Experiment 1 suggested that mental rotation was a piecemeal process. This conflicts with the results of Cooper and Podgorny (1976), who showed that rotation was a holistic process. This contradiction may stem from the fact that Cooper and Podgorny used random shapes as their stimuli. Such shapes are meaningless and, thus, may entail a different representational format and subsequent processing than that of meaningful objects. Previous research (e.g., Donnelly, Humphreys, \& Sawyer, 1994; Suzuki \& Cavanagh, 1995), however, suggests that meaningless objects entail a more segment-by-segment processing. The following experiment directly examines this question. Following the paradigm of Experiment 1, the pattern of errors was compared in rotating simple and complex meaningless stimuli.

\section{Method}

The method of Experiment 2 was identical in all respects to that of Experiment 1, except for the set of stimuli used and the participants. Only those aspects of the experiment that differ from those in Experiment 1 are specified below.

Participants. The participants were 28 undergraduate students. There were 8 males and 20 females, who ranged in age between 18 and 49 (mean age, 22.5). All the participants had normal or correctedto-normal vision and were right-handed. None of the participants had any experience with the stimuli or task prior to the experiment.

Materials. The sets of simple and complex meaningless objects were designed to be as equivalent as possible to the meaningful objects, except that these objects had to be meaningless. To achieve this, the meaningless objects were constructed by taking the set of meaningful objects and reorganizing their parts (see Figure 1 for examples). A pretesting study validated the meaningfulness of the entire set of stimuli. The meaningless set of stimuli matched the complexities of the meaningful set of stimuli used in Experiment 1. The compactness value produced for the meaningless stimuli were significantly different for the simple and complex stimuli. The complex stimuli had a mean value of $0.20(S D=0.02$, range, $0.16-0.21)$, and the simple stimuli had a mean value of $0.45(S D=$ 0.25 , range, 0.24-0.94) $[t(10)=2.41, p<.05]$. Furthermore, the compactness value for the meaningful and meaningless complex stimuli (Experiments 1 and 2) were not significantly different from one another $[t(10)=2.11, p>.05]$, and, similarly, the simple meaningful and meaningless objects were not significantly different $[t(10)=.35, p>.05]$.

\section{Results}

The data were were submitted to ANOVA. As in Experiment 1 , the data of interest were error rates. Once again, stimulus complexity (simple and complex) and angle of rotation $\left(0^{\circ}, 50^{\circ}, 50^{\circ}, 100^{\circ}\right)$ were used as within factors in the analyses.

As is illustrated in Figure 2 (right panel), there was no interaction between the angle of rotation and stimulus complexity $(F<1)$. This reflects the fact that the difference in error rates between the simple and complex stimuli remained consistent across the various angular rotations. There was a main effect of angle of rotation $[F(3,81)=$ $30.43, p<.01]$ - that is, the participants made more errors with greater angle of rotation. As with the meaning- 
ful objects, both the simple $[F(3,27)=14.67, p<.001]$ and the complex $[F(3,27)=14.02, p<.001]$ objects showed an increase in errors with increasing angle of rotation. Finally, there was also a main effect of complexity $[F(1,27)=$ $40.48, p<.01]$. There were more errors made with complex objects than with simple objects. There was no main effect of order of task $(F<1) .^{2}$

As in Experiment 1, the results with the error rates were confirmed by using $d^{\prime}$ values. There was a main effect of angle of rotation $[F(3,81)=61.62, p<.001]$, but no significant interaction between angle of rotation and complexity $(F<1)$. There was also a main effect of complexity $[F(3,27)=31.52, p<.001]$.

The analyses with the error rates were repeated with the response times to ensure that a speed-accuracy tradeoff was not present. There was a main effect of angle $[F(3,81)=23.21, p<.001]$. As with the error rates, this was found for both simple $[F(3,27)=14.01, p<.001]$ and complex objects $[F(3,27)=16.51, p<.001]$. Although this was only significant between $0^{\circ}$ and $50^{\circ}$ [complex: $t(27)=3.92, p<.001$; simple: $t(27)=4.78, p<$ $.001]$, the longest response time was again at $150^{\circ}$. There was a main effect of complexity $[F(1,27)=6.34, p<.05]$, with the complex objects requiring more time to process than did the simple objects. There was no interaction between angle and complexity $[F(3,81)=1.83, p>.05]$.

The study times of the complex and simple objects were compared. There was no significant difference between the study times for the complex and the simple stimuli $[t(27)=1.71, p>.05]$.

\section{Discussion}

In this experiment, we examined the type of transformations used when meaningless objects were mentally rotated. The increase in error rates as the angle of rotation increased indicates that the participants were engaging in more visual mental rotation processing in order to perform the task with greater angular rotations.

The main finding in contrast to the results of Experiment 1 , was that there was no interaction between the complexity of the objects and the angle of rotation. This was not predicted by a segment-by-segment processing model and is therefore consistent with a holistic process being used. The main effect of complexity was held constant across all angles of rotations. This may reflect the difficulty in encoding the stimuli, making the comparison, or some other factor that is not related to the rotation process per se. The result of this is a main effect of complexity, but no interaction with the angle of rotation.

The results of this study suggest that the findings of Cooper and Podgorny (1976) may have been a consequence of the type of stimuli they used. Specifically, meaningless objects result in holistic processing, and meaningful objects result in piecemeal processing. These results do not coincide with those of Donnelley et al. (1994) and Suzuki and Cavanagh (1995). This may be due to how the meaninglessness of the stimuli is operationalized. Other studies achieve meaningless stimuli by scrambling meaningful objects in such a way that maintains the meaningful components, but in a different spatial layout (e.g., a nonface has a mouth, eyes, nose, and so forth, but not in the same configuration as a real face). The stimuli in this experiment were made in such a way that the meaningless objects did not appear as scrambled meaningful objects (see Figure 1 for examples), and pretesting was conducted to make sure that the meaningless objects were not associated with a meaningful object.

The meaningless objects used here required that the participants had no general familiarity or experience with them. Hence, the difference in results across the two conditions could be explained by familiarity with the stimuli rather than by their meaning. Bethell-Fox and Shepard (1988) found that familiarity could affect the rotation process, in that practice at rotating the stimuli encouraged a more holistic representation. A third experiment addressed this by assessing the role of familiarity.

\section{EXPERIMENT 3 Familiar, Meaningless Objects}

The stimuli use in Experiment 1 were meaningful and, as such, they were also familiar relative to the meaningless stimuli used in Experiment 2. Hence, rather than meaningfulness accounting for the data, familiarity could be put forward as an explanation for the findings. In the third experiment, this possibility is addressed; prior to the rotation task, the participants were trained to make the meaningless stimuli familiar. Therefore, this experiment repeated the previous one, except that although the objects were still meaningless, they were now familiar to the participants.

\section{Method}

The method of Experiment 3 was identical to that of Experiment 2, except for the pretraining familiarization tasks that were given prior to performing the rotation task. The pretraining task was aimed at familiarizing the participants with the objects but not with the rotation task itself. Only those aspects of the experiment that differ from those in Experiment 2 are described below.

Participants. The participants were 28 undergraduate students. There were 7 males and 21 females, who ranged in age from 18 to 43 (mean age, 21.3). All the participants had normal or corrected-tonormal vision and were right-handed. None of the participants had any experience with the stimuli or task prior to the experiment.

Procedure. The participants performed an identical rotation task to that performed by the participants in Experiment 2; however, prior to this, they performed three familiarity tasks. The familiarization tasks were administered twice. On the first occasion, they were presented in isolation at least 1 day, but not more than 7 days, before the rotation task. On the second occasion, they were immediately followed by the rotation task. The tasks were chosen to provide experience and familiarity with the upright stimuli but not with the rotation task. The tasks required imaging the objects and rating how vivid the image was, memorizing the objects, and comparing them with other objects.

Following the experiment, the participants were asked to complete a number of stimulus assessment ratings to allow validation of the efficiency of the procedure.

\section{Results}

The data were submitted to an ANOVA. As before, the data of interest were error rates; stimulus complexity (sim- 
ple and complex $)$ and angle of rotation $\left(0^{\circ}, 50^{\circ}, 100^{\circ}\right.$, and $150^{\circ}$ ) were used as within factors in the analyses.

As is illustrated in Figure 3, there was no interaction between the angle of rotation and stimulus complexity $(F<1)$, reflecting that the complexity of the stimulus had no effect on changes in error rate with greater angles of rotation. There was a main effect of angle of rotation $[F(3,81)=76.2, p<.001]$; that is, the participants made more errors with greater angles of rotation. This was found for both the simple objects $[F(3,27)=55.55, p<.001]$ and the complex objects $[F(3,27)=36.81, p<.001]$. There was also a main effect of complexity $[F(1,27)=$ $86.03, p<.001$ ], with complex objects being more prone to errors than were simple objects. There was no main effect of order $(F<1) .^{3}$

The results with the error rates were confirmed by using $d^{\prime}$ values. There was a main effect of angle of rotation $[F(3,81)=108.95, p<.001]$, no significant interaction between angle of rotation and complexity $(F<1)$, and a main effect of complexity $[F(3,27)=93.49, p<.001]$.

As before, further analyses were performed using response time data. There was a main effect of angle $[F(3,81)=23.21, p<.001]$. The increase in response times was found for both the simple objects $[F(3,27)=$ $11.49, p<.001]$ and the complex objects $[F(3,27)=$ $9.61, p<.001]$. Again, the longest response time was at $150^{\circ}$, although the only significant increase was between $0^{\circ}$ and $50^{\circ}$ [complex: $t(27)=3.92, p<.001$; simple: $t(27)=4.78, p<.001]$. There was no interaction be-

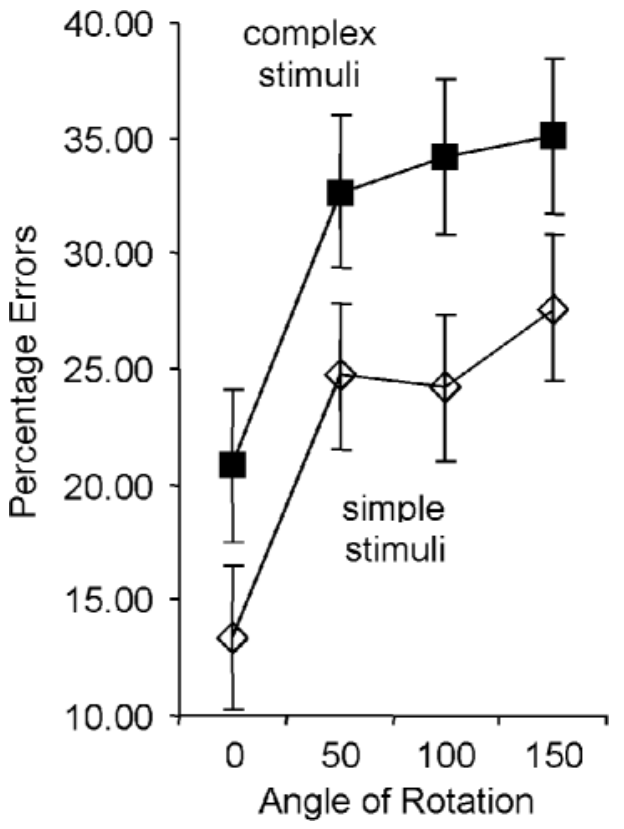

Figure 3. Error rate data for mental rotation of familiar, meaningless objects. The slope of increased errors with greater angular rotation for the simple and complex objects was identical, consistent with rotation using a holistic process. tween angle and complexity $(F<1)$ and no main effect of complexity $[F(1,27)=3.18, p>.05]$.

Study times of the complex and simple objects were compared. The participants took longer to study the complex objects (mean time 1,587, $S D=698 \mathrm{msec}$ ) than the simple objects (mean time $1,354, S D=468 \mathrm{msec})[t(27)=$ $2.15, p<.05]$.

To ensure that the pretraining familiarity tasks achieved their goal, the results from the pretraining tasks were examined. First, the three computer-presented tasks were analyzed.Error rates were minimal, as planned-the pretraining was designed only to achieve familiarity. The response times on the first occasion were compared with those of the second occasion for the comparison and memory tasks. In both cases, there was a reduction in response times for correct answers from the first to the second time [comparison task: $t(27)=3.53, p<.01$; memory task: $t(27)=3.55, p<.01]$. The tasks were influencing the participants' performance, enabling them to provide an accurate answer in a shorter amount of time. For the vividness task, there was an increase in the ratings of vividness of the image generated from the first to the second time $[t(27)=6.35, p<.001]$.

In addition, the participants' judgments about the stimuli were compared with those from the original stimulus assessment, performed by a different group of participants. This revealed that the meaningless stimuli used in this experiment with pretraining were rated as being as familiar as the meaningful stimuli in Experiment 2.

\section{Discussion}

In this experiment, pretraining with the meaningless stimuli increased familiarity, enabling the roles of meaning and familiarity to be examined separately. Although increased familiarity resulted in an overall reduction of errors, the pattern of errors remained unchanged. This lack of an interaction between angle of rotation and complexity suggests that the results found in Experiments 1 and 2 were due to the meaning (or lack of meaning) of the objects rather than to their familiarity.

\section{GENERAL DISCUSSION}

The literature on visual mental rotation presents opposing views about whether the transformation encompasses a holistic process, whereby the image is rotated as a single image, or a piecemeal process, whereby the image is rotated segment by segment and reconstructed to form a whole image. Past research has tried to address this question indirectly by using images of impossible objects (Dror et al., 1997) and hierarchical images of large letters composed of smaller letters (e.g., Paquet, 1991; Robertson $\&$ Palmer, 1983). However, most of the research has addressed this issue by comparing the rate of rotation of stimuli of different complexities. The logic has been that if a holistic process were used, the complexity of the stim- 
uli would not affect the rate of rotation, since the image would be a single entity within a rotated buffer. In contrast, if a piecemeal process were used, the complexity of the image would encompass different rates of rotation, as the image is rotated segment by segment, and more complex images have more segments to be rotated. This line of research has produced conflicting results. Some researchers have not found an effect of complexity and have concluded that mental rotation is a holistic process (e.g., Cooper \& Podgorny, 1976). Other researchers have found such an effect and have concluded that rotation is a piecemeal process (e.g., Folk \& Luce, 1987; Yuille \& Steiger, 1982).

The research reported here differed from the previous research in two main respects. First, we investigated whether the format of representation of the stimuli determined whether the rotation would be holistic or piecemealspecifically, whether the meaningfulness (or lack of it) of the stimuli would entail different mental processing. Second, the pattern of error rates was used to distinguish between holistic and piecemeal processing. This followed logic similar to that for response times but had the advantage that error rates allowed a differentiation between holistic processing and parallel piecemeal processing.

The results show that when the images were of meaningful objects, there was an interaction between angle of rotation and complexity, as predicted in a piecemeal rotation. When the objects were meaningless, there was no interaction. This finding can be attributed to the meaning of the objects. First, the alternative account for the data that attributes the pattern of results to the familiarity inherent in meaningful objects was excluded. Pretraining on the meaningless objects increased familiarity with them, but the pattern of results continued to show no interaction, consistent with holistic rotation. Second, the meaningless objects were not formed by scrambling the location of meaningful components (like the arrangement of facial features; e.g., Donnelley et al., 1994; Suzuki \& Cavanagh, 1995) but by rearranging the parts so that there were no meaningful components. This was to make sure that the new meaningless object did not appear as a distorted meaningful object. Hence, the data were consistent with the meaningful objects' being rotated via a piecemeal process, whereas the data from the meaningless objects were consistent with rotation through a holistic process.

Bethell-Fox and Shepard (1988) supported the idea that mental rotation could be either a piecemeal or holistic process. They suggested that greater familiarity with the stimuli would change the rotation process from a piecemeal to a holistic process. The findings in this study do not support this conjecture; although familiarity enabled fewer errors overall, the pattern of errors across the conditions remained the same. Bethell-Fox and Shepard achieved familiarity through exposure to the stimuli in the context of the rotation task. Familiarity with the rotation task itself, therefore, could be different from familiarity with the stimuli per se.

These results suggest that the transformations during mental rotations were not fixed in one format-either holistic or piecemeal. Rather, mental rotation is a flexible process, with both holistic and piecemeal transformations' being possible. This study suggests that one of the factors that could determine the type of processing taking place in mental rotation, and, perhaps, other cognitive processes, is the meaningfulness of the stimuli. One way in which meaning could influence the process would be if meaning were an important factor in constructing the representation. If the format of the representation played a role in how it was processed, factors affecting the initial format of the representation would affect the type of process.

It would be relatively easy to employ a piecemeal rotation process on a representation formed of segments. In contrast, if the segments are not "natural" entities, it may be easier to rotate the entire image in a holistic fashion. There is some support for this claim; meaningful objects are represented by their constituting segments and their spatial relations to allow abstract viewpoint-invariantrepresentations (e.g., Biederman, 1987; Biederman \& Gerhardstein, 1993; Cave \& Kosslyn, 1993).

The availability of both piecemeal and holistic mental rotation processing can enable flexibility; this maximizes the accurate processing of information, in the face of constraints imposed by the circumstances (see also Cohen \& Kubovy, 1993). A central issue for research is to understand how and why certain factors affect cognitive processing the way they do. The findings here allow us to take a further step in this direction and to speculate on some possibilities.

Piecemeal processing may be more flexible than holistic processing. Piecemeal processing appears to allow a wider variety of transformations (e.g., folding). Holistic processing may impair such transformations, because all the information is contained as a single undifferentiated unit, whereas piecemeal processes may facilitate it through allowing individual units to separate and recombine in a variety of ways. However, although piecemeal processing may be more flexible, holistic processing may be "safer" and less computationally demanding. It is safer in the sense that spatial information is more easily preserved and remains relatively intact throughout the process, rather than being reconstructed. It is less demanding since it requires fewer processing steps (parsing the image to its constituting segments - if it is not already represented in this format-and rotating and dealing with each segment separately and then reconstructing the segments to form a whole image). Therefore, even though piecemeal processing might be more flexible, if there are no representations or task demands to re-reinforce its use, holistic processing may be invoked if it is more accurate and less computationally demanding.

A note of caution needs to be introduced before the results are interpreted conclusively as demonstrating different processes during rotation. Most of the effect of the angle of rotation that was present was between the angles of $0^{\circ}$ and $50^{\circ}$. Although this does not exclude the occurrence of rotation, it does not provide unequivocal confirmation. One possibility is that the deadline procedure made the rotation at higher angles very difficult and that the partic- 
ipants were guessing on a proportion of the trials. However, even if this were the case, the interaction between angle of rotation and complexity would not be explained by this guesswork at higher angles.

If the participants were guessing when the angles of rotation were higher, this would lead to the prediction that performance would become less accurate than expected as the angle of rotation increased. This was not noticeable in the results; if the errors at the lower angles are extrapolated to the higher angles, it can be seen that accuracy was actually better than expected at these angles. The plateau could also be explained if a floor effect was being produced - that is, the participants were performing at chance at the greater angles of rotation. This was not the case, either; for example, in the meaningful stimuli, error rates at the greatest angle of rotation averaged $27 \%$ in the simple stimuli and $32 \%$ in the complex stimuli, well below the chance level of $50 \%$.

A further possibility, therefore, is that the strategy changed when the angle of rotation was high and the rotation task became very difficult. Instead of rotating the image and matching the whole representation, the participant may have used a viewpoint-independent strategy. This would probably involve their looking for a diagnostic part of the shape and using this to provide the response. However, the only diagnostic part of the shape was the modification, or, in the case of the same trials, an absence of a modification. The task, therefore, might encourage a viewpoint-independent strategy, but the differing locations of the modification might have made this strategy difficult to use. Similar findings to the ones described here have been described in other studies, notably, for example, in such studies as those carried out by Lawson and Jolicœur (1998), in which participants had to perform an identification of a briefly presented stimulus. In their experiment, unlike in the present study, there was no deadline procedure or pressure to respond, but, similar to the present study, the stimulus was only presented for a very brief time. Lawson and Jolicœur suggested that a view-specific code was used to identify the stimulus, using a transformation-followed-by-matchstrategy. This was adequate, in their experiment, for angles of up to $60^{\circ}$. However, if the presentation time of the stimulus prevented this strategy at higher angles, the participant had to change to a view-independentstrategy. This has been used to explain similar findings in other naming studies (e.g., Jolicœur \& Milliken, 1989; Murray, 1995). A viewpoint-independent computational model of object recognition also showed a similar function across angle of rotation (Hummel \& Biederman, 1992).

An alternative possibility for overcoming the demands of the brief presentation time is that only part of the stimulus was rotated, giving the appearance of a holistic rotation. If the participant rotated the part containing the modification, this strategy would be successful. However, neither this model for the processing of the stimuli at the higher levels nor the previous model satisfactorily explains the interaction between the angle of rotation and the complexity that was found at the lower angles of rotation.
The research reported here stresses the possible link between representations and their subsequent processing. The meaningfulness of objects has been shown in this study to be a potential factor in determining their representations, which may in turn influence whether a piecemeal or holistic rotation process will be used subsequently. The explanations of why and how meaningfulness entails one rotation process and not the other process should also influence how such objects will be processed in a variety of other cognitive domains. Similarly, other factors could prove critical in influencing the format and processing of representations - clear topics for further research.

\section{REFERENCES}

Ashworth, A., \& Dror, I. E. (2000). Object identification as a function of discriminability and learning presentations: The effect of stimulus similarity and canonical frame alignment on aircraft identification. Journal of Experimental Psychology: Applied, 6, 148-157.

Attneave, F. (1957). Physical determinants of the judged complexity of objects. Journal of Experimental Psychology, 53, 221-227.

Bethell-Fox, C. E., \& Shepard, R. N. (1988). Mental rotation: Effects of stimulus complexity and familiarity. Journal of Experimental Psychology: Human Perception \& Performance, 14, 12-23.

BIEDERMAN, I. (1987). Recognition-by-components: A theory of human image understanding. Psychological Review, 94, 115-147.

Biederman, I., \& Gerhardstein, P. C. (1993). Recognizing depthrotated objects: Evidence and conditions for three-dimensional viewpoint invariance. Journal of Experimental Psychology: Human Perception \& Performance, 19, 1162-1182.

Cave, C. B., \& Kosslyn, S. M. (1993). The role of parts and spatial relations in object identification. Perception, 22, 229-248.

Cedrus Cor Poration (1991). Superlab: General purpose testing package (Version 1.4 Macintosh Edition) [computer software]. San Pedro, CA: Author.

Cerella,J., Poon, L. W., \& Fozard, J. L. (1981). Mental rotation and age reconsidered. Journal of Gerontology, 36, 620-624.

Cohen, D., \& Kubovy, M. (1993). Mental rotation, mental representation, and flat slopes. Cognitive Psychology, 25, 351-382.

Cooper, L. A., \& Podgorny,P. (1976). Mental transformations and visual comparison processes: Effects of complexity and similarity. Journal of Experimental Psychology: Human Perception \& Performance, 2, 503-514.

Donnelly, N., Humphreys, G. W., \& Sawyer, J. (1994). Stimulus factors affecting the categorisation of faces and scrambled faces. Acta Psychologica, 85, 219-234.

Dror, I. E., \& Gallogly, D. P. (1999). Computational analyses in cognitive neuroscience: In defense of biological implausibility. Psychonomic Bulletin \& Review, 6, 173-182.

Dror, I. E, Ivey, C., \& Rogus, C. (1997). Visual mental rotation of possible and impossible objects. Psychonomic Bulletin \& Review, 4, 242-247.

Dror, I. E., \& Kosslyn, S. M. (1994). Mental imagery and aging. Psychology \& Aging, 9, 90-102.

Dror, I. E., ZAGAESKI, M., \& Moss, C. F. (1995). Three-dimensional target recognition via sonar: A neural network model. Neural Networks, $\mathbf{8}$, 149-160.

FolK, M. D., \& LUCE, R. D. (1987). Effects of stimulus complexity on mental rotation rate of polygons. Journal of Experimental Psychology: Human Perception \& Performance, 13, 395-404.

Hummel, J. E., \& Biederman, I. (1992). Dynamic binding in a neural network for shape recognition. Psychological Review, 99, 480-517.

Joliceur, P., \& Milliken, B. (1989). Identification of disoriented objects: Effects of context of prior presentations. Journal of Experimental Psychology: Learning, Memory, \& Cognition, 15, 200-210.

Kosslyn, S. M. (1981). The medium and the message in mental imagery: A theory. Psychological Review, 88, 46-66.

LAWSON, R, \& Jolicceur, P. (1998). The effects of plane rotation on the recognition of brief masked pictures of familiar objects. Memory \& Cognition, 26, 791-803. 
MArr, D. (1982). Vision. San Francisco: Freeman.

Murray, J. E. (1995). Imagining and naming rotated natural objects. Psychonomic Bulletin \& Review, 2, 239-243.

MurRay, J. E. (1997). Flipping and spinning: Spatial transformation procedures in the identification of rotated natural objects. Memory \& Cognition, 25, 96-105.

PAQUeT, L. (1991). Mental rotation of compound stimuli: The effects of task demands, practice, and figural goodness. Memory \& Cognition, 19, 558-567.

Podgorny, P., \& ShePard, R. N. (1983). Distribution of visual attention over space. Journal of Experimental Psychology: Human Perception \& Performance, 9, 380-393.

Robertson, L. C., \& Palmer, S. E. (1983). Holistic processes in the perception and transformation of disoriented figures. Journal of Experimental Psychology: Human Perception \& Performance, 9, 203-214.

Salthouse, T. A. (1992). Shifting level of analysis in the investigation of cognitive aging. Human Development, 35, 321-341.

Schacter, D. L., Cooper, L. A., \& Delaney, S. M. (1990). Implicit memory for unfamiliar objects depends on access to structural descriptions. Journal of Experimental Psychology: General, 119, 5-24.

Schacter, D. L., Cooper, L. A., Delaney, S. M., Peterson, M. A., \& Tharan, M. (1991). Implicit memory for possible and impossible objects: Constraints on the construction of structural descriptions. Journal of Experimental Psychology: Learning, Memory, \& Cognition, 17, 3-19.

Suzuki, S., \& CAVANAgh, P. (1995). Facial organization blocks access to low-level features: An object inferiority effect. Journal of Experimental Psychology: Human Perception \& Performance, 21, 901-913.

Verso, J. (1988). Picture it in cross stitch. Devon, U.K.: David \& Charles.
Yuille, J. C., \& Steiger, J. H. (1982). Nonholistic processing in mental rotation: Some suggestive evidence. Perception \& Psychophysics, 31, 201-209.

\section{NOTES}

1. In addition, the data from the meaningful stimuli were analyzed using a square-root transformation of the original raw error rates. This addressed the potential problems of the distribution of the data. The analyses confirmed those with the raw data: There was a main effect of angle of rotation $[F(3,81)=22.19, p<.001]$, a significant interaction between angle of rotation and complexity $[F(3,81)=8.27, p<.01]$, and a main effect of complexity $[F(3,27)=5.10, p<.05]$.

2 . An analysis of the square-root transformation of the error rates from the meaningless stimuli gave the same pattern of errors as that with the raw data. There was a main effect of angle of rotation $[F(3,81)=27.61$, $p<.001]$, no significant interaction between angle of rotation and complexity $(F<1)$, and a main effect of complexity $[F(3,27)=44.61, p<$ $.001]$.

3. The pattern of results found with the raw data from the meaningless, familiar stimuli was further confirmed with a square root transformation. There was a main effect of angle of rotation $[F(3,81)=$ $90.46, p<.001]$, no significant interaction between angle of rotation and complexity $[F(3,81)=1.18, p>.05]$, and a main effect of complexity $[F(3,27)=84.07, p<.001]$.

(Manuscript received June 25, 1998; revision accepted for publication February 9, 2001.) 